\title{
Pupil Detection Execution Time Reduction in Iris Recognition System
} Maha A. Hasso

College of Computer Sciences and mathematics

Bayez K. Al-Sulaifanie

College of Electronic Eng.
Kaydar M. Quboa

College of Electronic Eng.

Received on: 02/03/2008

University of Mosul, Iraq

Accepted on: 11/06/2008

\section{ABSTRACT}

Iris recognition is regarded as the most reliable and accurate biometric identification system available. The work presented in this paper involves improving iris segmentation to reduce execution time. To determine the performance of the iris system two databases of digitized grayscale eye images are used.

The segmentation process in the iris recognition system is used to localize the circular iris and pupil regions, excluding eyelids and eyelashes. New techniques are proposed and implemented for pupil detection. These techniques are mask, profile and the combined profile mask (CPM) technique. The extracted iris region is normalized into a rectangular block with constant dimensions to account for imaging inconsistencies.

The feature extraction technique is based on 2D Gabor filters. The Hamming distance is used to classify the iris templates, and the FAR, FRR and RR are calculated.

The results of the study proved that the best technique for pupil detection is when using the combined technique. It gives about $100 \%$ success rate for pupil detection.

Keywords: Iris recognition, iris, 2D Gabor filter, Hamming distance method.

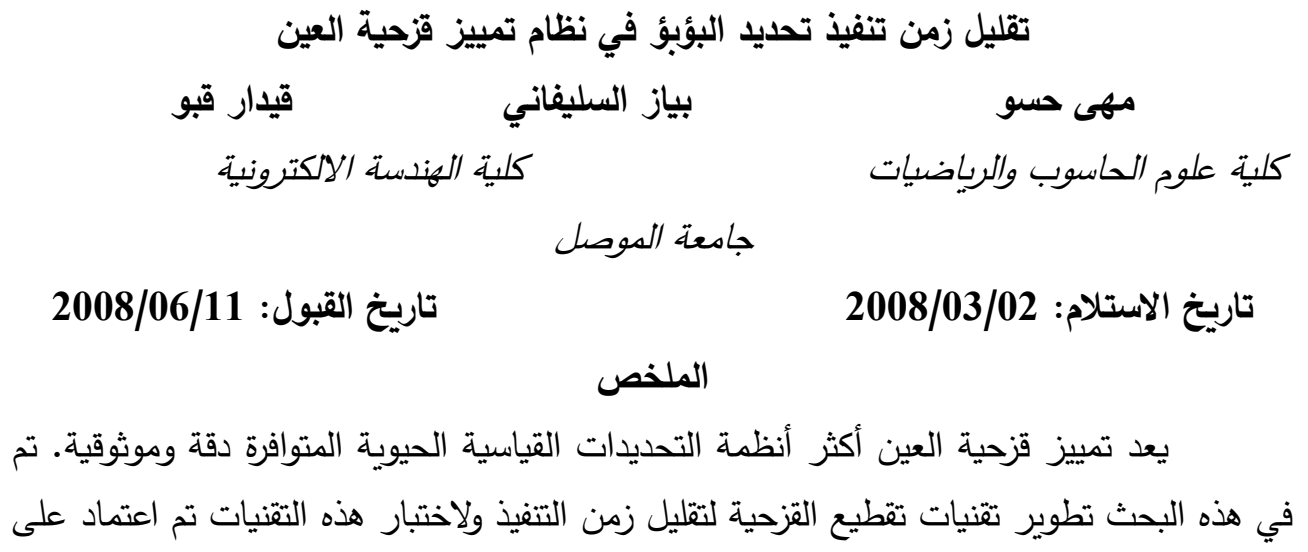




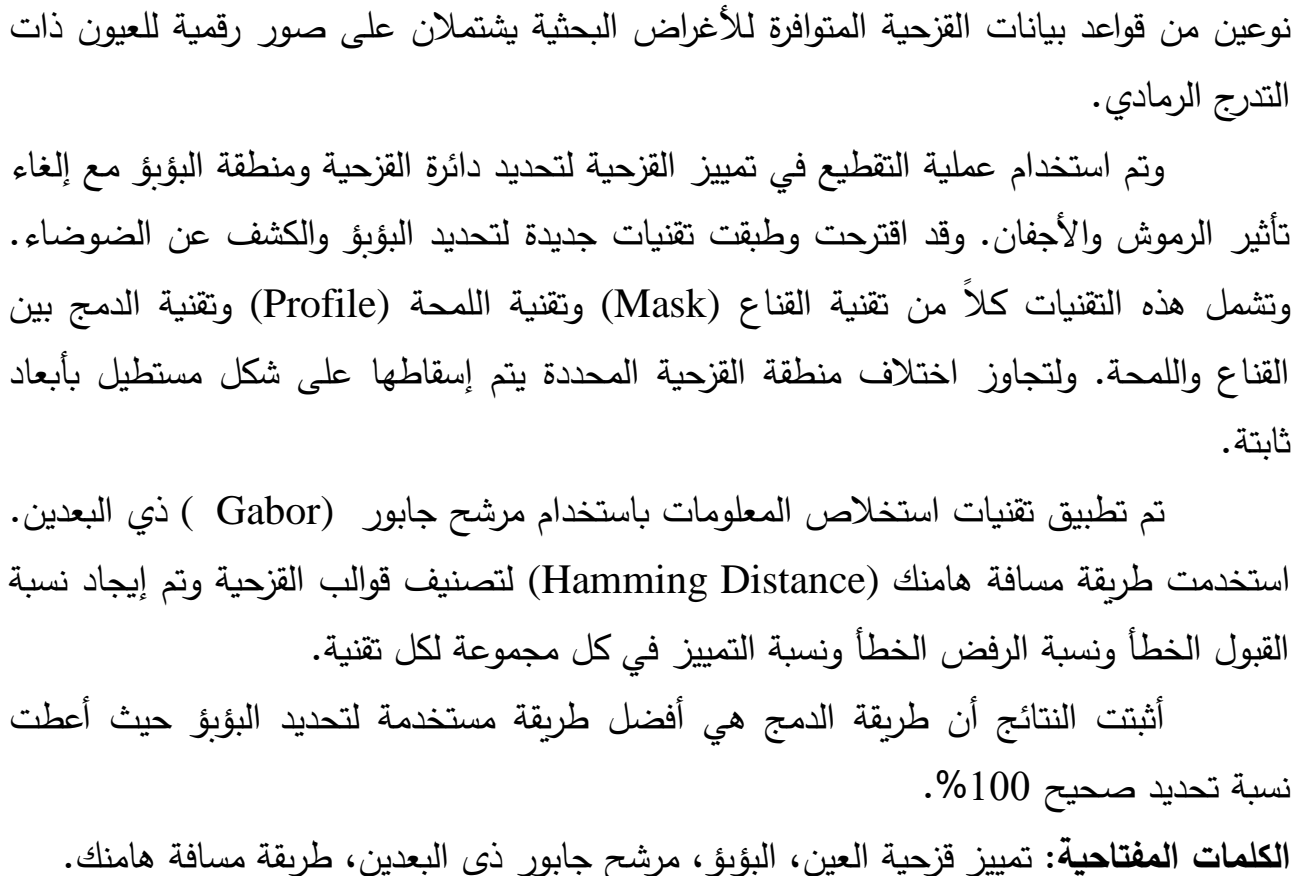

\section{Introduction}

Iris recognition systems produce much less errors than any other known and applied biometric technology. Obviously, it is necessary to find parts of the human body that offer stable, very unique, easy measurable and fast processable patterns in order to increase quality and speed of identification algorithms [1].

The iris, as shown in Figure (1), is a physiological biometric feature. It contains unique texture and is complex enough to be used as a biometric signature [2].

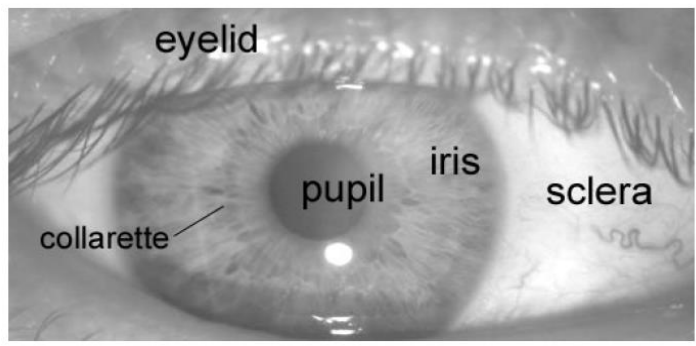

Figure (1): Physiological biometric feature, a front-on view of the human iris [2].

Compared with other biometric features such as face and fingerprint, iris patterns are more stable and reliable. It is a unique character in each 
person and stable with age. Furthermore, iris recognition systems can be non-invasive to their user [3][4].

The best-known and most widely iris recognition algorithm is that of Daugman [3], [5] and [6]. The Daugman algorithm first locates the pupillary and limbic boundaries of the iris using an integro-differential operator that finds the circles in the image where the intensity is changing most rapidly with respect to changes in the radius [5].

Another well-known is due to Wildes [4]. The Wildes algorithm located the iris boundaries by creating a binary edge map using gradientbased edge detection, and then found the centers and radii of these circles via a Hough transform. The upper and lower eyelids are located similarly using parabolic arcs [4].

Boles and Boashash [7] proposed an algorithm that located the pupil center using an edge detection method, recorded gray level values on virtual concentric circles, and then constructed the zero-crossing representation on these virtual circles based on a one-dimensional dyadic wavelet transform.

\section{Iris recognition system}

Generally iris recognition system is composed of many stages as shown in Figure (2).

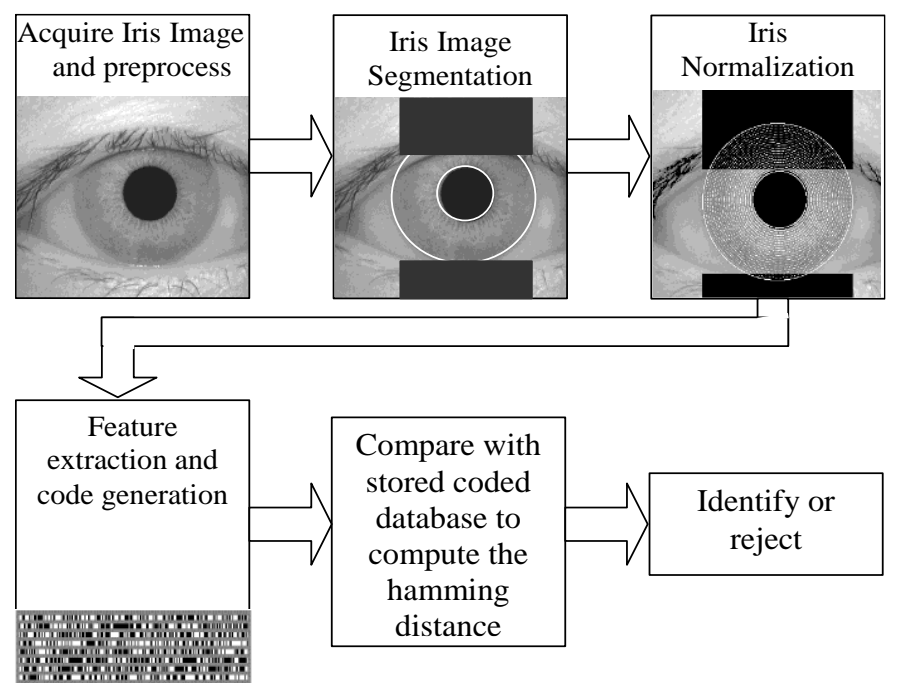

Figure (2): Block diagram of an Iris Recognition System

The interactions and dependencies between these stages are considered and the interplays are explored between iris sensor design, accuracy, efficiency as well as user friendliness and acceptability of iris recognition systems [8]. 


\subsection{Segmentation}

The segmentation of the iris region is to localize the actual iris region in a digital eye image. Two circles can approximate the iris region, one for the iris/sclera boundary and another, interior to the first, for the iris/pupil boundary. The eyelids and eyelashes normally occlude the upper and lower parts of the iris region. Also, specular reflections can occur within the iris region corrupting the iris pattern [9]. A technique is required to isolate and exclude these artifacts as well as locate the circular iris regions .

\subsubsection{Pupil Detection Using Circular Hough Transform}

The circular Hough transform can be employed to deduce the radius and center coordinates of the pupil and iris regions. Hough space parameters are the center coordinates $\mathrm{xc}$ and $\mathrm{yc}$, and the radius $\mathrm{r}$, which are able to define any circle according to the following equation:

$$
\mathrm{x}_{\mathrm{c}}^{2}+\mathrm{y}_{\mathrm{c}}^{2}-\mathrm{r}^{2}=\mathrm{O}
$$

A maximum point in the Hough space will correspond to the radius and center coordinates of the best circle defined by the edge points. The parabolic Hough transform was used to detect the eyelids.

The pupil always lies in a dark area in the iris region, therefore novel techniques are proposed using mask, profile and combination between them for pupil detection. These techniques give high accurate and reduce the execution time required to detect the pupil.

Basically, the image has unadvisable space. This unadvisable space could be reduced by segmenting the eye image into $16,(4 \times 4)$ segments. The used search space is the inner four segments as shown in Figure (3). This reduced search space is used in the next pupil detection steps [10].

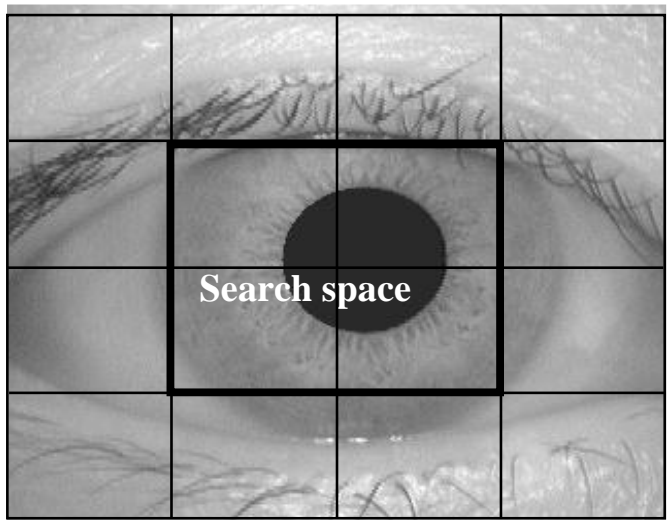

Figure (3): The reduced search space in the pupil detection. 


\subsubsection{Pupil Detection Using Mask}

After segmentation to reduce search space, the convolution process is applied using a mask of $(2 \times 2)$ with ones coefficients. The result of the average masked points in an image is stored in a vector and reduced by threshold. The indices of minimum and maximum values in the reduced vector are considered to be the starting and ending coordinates of the pupil as shown in Figure (4) [10].

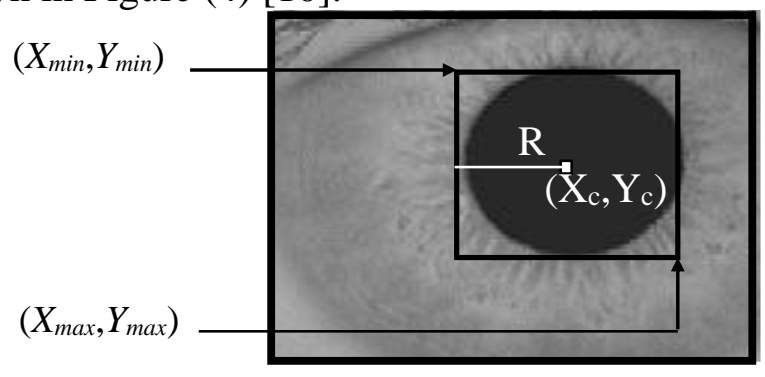

Figure (4): The pupil detection by mask, implemented on an image.

The radius of pupil $(\mathrm{R})$ is calculated as the average of two radii (R1), and (R2). The radius (R1) is calculated from the values of Xmax and Xmin according to the following equation:

$\mathrm{R}_{1}=\frac{\mathrm{X}_{\max }-\mathrm{X}_{\min }}{2}$

while the radius (R2) is calculated from the values of Ymax and Ymin according to the following equation:

$\mathrm{R}_{2}=\frac{\mathrm{Y}_{\max }-\mathrm{Y}_{\min }}{2}$

Hence the radius of the pupil is calculated in accordance to the following equation:

$\mathrm{R}=\frac{\mathrm{R}_{1}+\mathrm{R}_{2}}{2}$

\subsubsection{Pupil Detection Using Profile}

The profile is a tool of an image processing techniques. The profile of an image region is a projection of compact representation in the spatial pixel content distribution and has been successfully employed in this work.

The pupil center is calculated by finding the maximum occurrence of an intensity value in the rows and columns [10]. The pupil lies on dark region; therefore the lowest intensity value has the maximum occurrence in the profile. The column index of the maximum occurrence represents the $\mathrm{X}$ coordinated of pupil center, denoted as Indcol. The row index of the maximum occurrence represents the $\mathrm{Y}$ coordinated of pupil center, denoted as Indrow. 
The radius of the pupil $(\mathrm{R})$ is calculated as the average of two calculaed radii (R1) and (R2). The radius $\mathrm{R} 1$ is calculated from the minimum and maximum values of row profile compared with threshold to determine the pupil region, denoted as RIndmin, RIndmax. Then the radius (R1) according to the following equation:

$\mathrm{R}_{1}=\frac{\mathrm{RInd}_{\text {max }}-\mathrm{RInd}_{\text {min }}}{2}$

While the radius $\mathrm{R} 2$ is calculated from the minimum and maximum values of column profile compared with threshold to determine the pupil region, denoted as CIndmin, CIndmax. Then the radius (R2) according to the following equation:

$\mathrm{R}_{2}=\frac{\mathrm{CInd}_{\max }-\mathrm{CInd}_{\text {min }}}{2}$

Hence the radius of the pupil and its center coordinates are calculated in accordance to the following equations:

$\mathrm{R}=\frac{\mathrm{R}_{1}+\mathrm{R}_{2}}{2}$

This method gives accurate pupil center detection. However this accuracy is reduced when measuring the pupil radius because of variability of threshold between samples.

\subsubsection{Combine Profile and Mask (CPM) for Pupil Detection}

It was decided to use the combine profile and mask (CPM) technique for pupil detection. This increases the accuracy for the two used databases. First, the profile is used to find the center coordinates then the mask is used to determine the radius [10].

\section{Results and Discussions}

The execution time of pupil detection using circular Hough transform for image samples from CASIA and Bath databases [11] [12] are carried out and the results are shown in Figure (5).

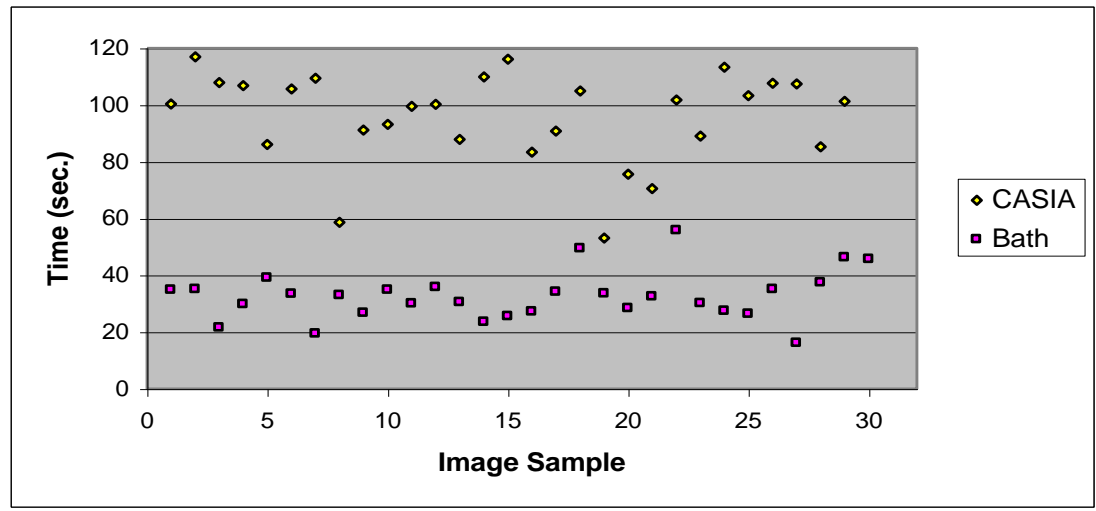


Figure (5): Execution time for pupil detection using circular Hough transforms for samples from CASIA and Bath databases.

It is clear from the Figure (5) that the execution time for CASIA database is in the range of (50-150) seconds, while for Bath it is around (2565) seconds. The execution time of pupil detection using the proposed techniques (mask, profile, and CPM technique) for the same samples of CASIA and Bath databases and the results are shown in Figure (6) and Figure (7).

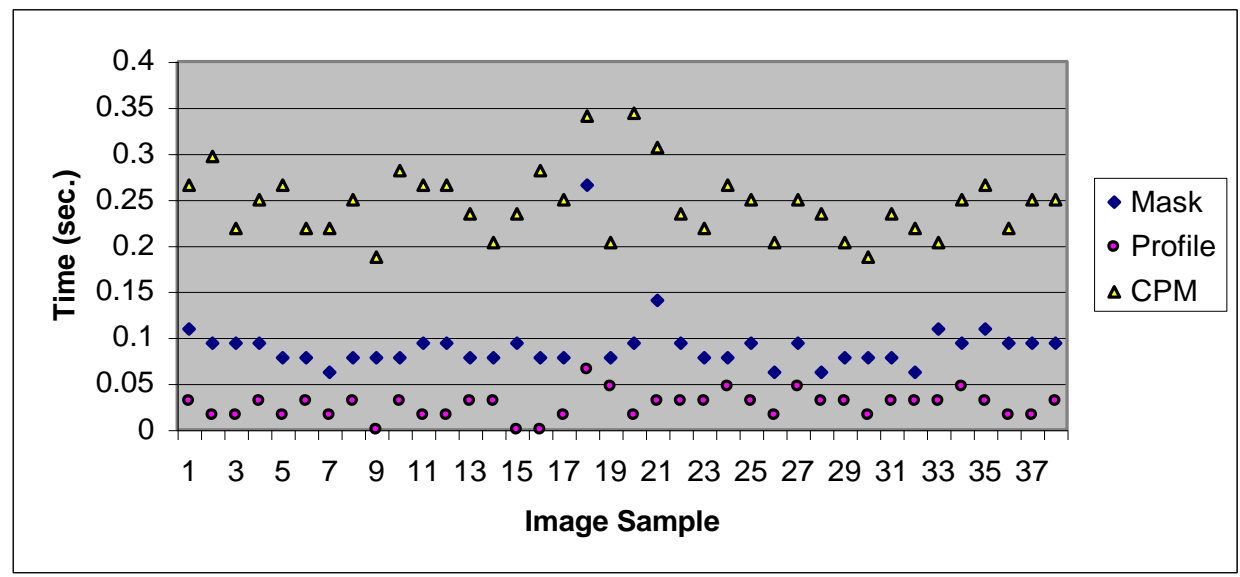

Figure (6): Execution time for pupil detection using new techniques for image samples from CASIA database.

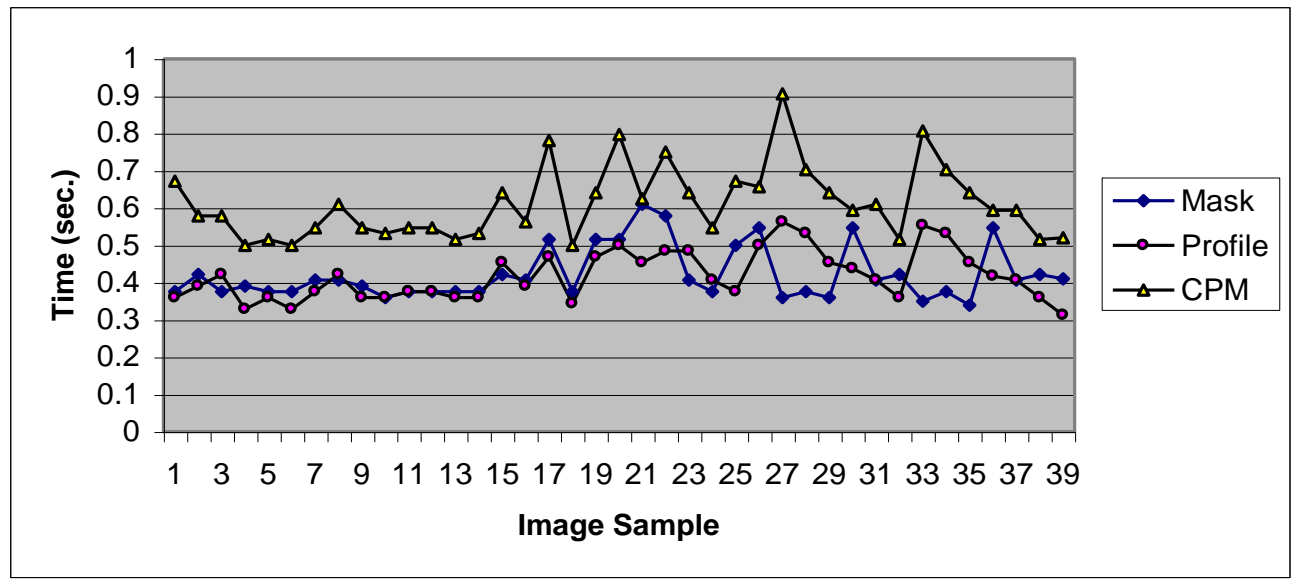

Figure (7): Execution time for pupil detection using new techniques for image samples from Bath database.

From Figures (6) and (7), it is clear that the execution time using mask, profile and CPM techniques is less than 0.4 seconds compared to 
around 110 seconds when circular Hough transform is applied to CASIA database samples while it is less than 0.9 seconds compared to around 50 seconds for Bath database samples.

Pupil detection is carried out for images of CASIA and Bath databases using circular Hough transform and the new proposed techniques mask, profile and CPM. To compare the results, the success rates is used as a measure for comparison. The obtained results are tabulated in Table (1).

Table (1): The success rate of pupil detection for different techniques

\begin{tabular}{|l|l||l|}
\hline Technique Database & $\begin{array}{l}\text { CASIA } \\
\%\end{array}$ & $\begin{array}{l}\text { Bath } \\
\%\end{array}$ \\
\hline Circular Hough Transform & 97.8 & 96.4 \\
\hline Mask & 100 & 95.3 \\
\hline Profile & 98.3 & 93.9 \\
\hline Combined Profile and Mask & 100 & 99.1 \\
\hline
\end{tabular}

From Table (1), the success rate using mask and the CPM techniques is $100 \%$ for CASIA database compared with $97.8 \%$ using circular Hough transform and $98.3 \%$ using profile technique, while it is $95.3 \%$ using mask and $99.1 \%$ using CPM for Bath database compared with $96.4 \%$ using circular Hough transform and $93.9 \%$ using profile technique. It is clear that the new proposed technique CPM is the best compared to the other.

The decision criterion for FAR, FRR and maximum RR are calculated and the results of the code size of 2048 and decision criterion of 0.36 will be the most suitable choice which gives a recognition rate of $98.8 \%$ with FAR of $0.0 \%$ and FRR of $1.16 \%$ for CASIA database. While the recognition rate is $100 \%$ for the Bath database.

\section{Conclusion}

It can be stated that segmentation is the critical stage of iris recognition, since areas that are wrongly identified as iris regions will corrupt biometric templates resulting in poor recognition. The success rate in pupil detection for the proposed technique reaches $100 \%$ in execution time of 5 seconds for the proposed mask and combine (CPM) techniques applied to CASIA database. Using CPM technique the success rate is $98.3 \%$ when applied to CASIA database. For Bath database the success rate is 99.1\% using CPM technique at execution time of about 0.9 seconds. The success rate for the CASIA database is $87.6 \%$ of the images managed to segment successfully due to poor imaging conditions, while it is $81 \%$ of the 
Bath database images segmented correctly. The best segmentation technique is when using CPM for pupil detection and circular Hough transform for iris detection.

\section{REFERENCES}

[1] Burghardt Tilo, (2002), "Inside Iris Recognition", Report on Identity Verification, Assignment Information Security, COMS40213, Master Course in Global Computing and Multimedia, University of Bristol.

[2] Zhu Yong, Tan Tieniu and Wang Yunhong, (2000), "Biometric Personal Identification Based on Iris Patterns", 15th International Conference on Pattern Recognition (ICPR).

[3] Daugman John G., (1993), "High Confidence Visual Recognition of Persons by a Test of Statistical Independence", IEEE Transaction on Pattern Analysis and Machine Intelligence, Vol. 15, no. 11, pp. 1148-1161.

[4] Wildes Richard P., (1997), "Iris Recognition: An Emerging Biometric Technology", Proceedings of the IEEE, Vol. 85, No. 9, pp. 1348-1363.

[5] Daugman John, (2004), "How Iris Recognition Works", IEEE Transactions on Circuits and Systems for Video Technology, Vol. 14, No. 1, pp. 21-30.

[6] Daugman J., (2003), "The Importance of Being Random: Statistical Principles of Iris Recognition", Pattern Recognition, Vol., 36, pp., 279-291.

[7] Boles W. W. and Boashash B., (1998), "A Human Identification Technique Using Images of the Iris and Wavelet Transform", IEEE Transactions on Signal Processing, Vol. 46, No. 4, pp. 1185-1188.

[8] Plemmons Robert, Horvatha Michael, Leonhardta Emily, Paucaa Paul, Prasadb Sudhakar, Robinsona Stephen, Settya Harsha, Torgersena Todd, Grachtc Joeseph van der, Dowskid Edward, Narayanswamyd Ramkumar and Silveirad Paulo E. X., (2004), "Computational Imaging Systems for Iris Recognition". Proceeding of SPIE Vol. 5559, Denver, pp. 346-357. 
[9] Libor Masek, (2003), "Recognition of Human Iris Patterns for Biometric Identification", Report Submitted Bachelor of Engineering Degree, School of Computer Science and Software Engineering, The University of Western Australia.

[10] Al-Gurairi A. H. Maha (2006), "Biometric Identification Based on Improved Iris Recognition Techniques", Ph. D. Thesis, College of Computer and Mathematical Sciences , University of Mosul , Iraq

[11] Chinese Academy of Sciences-Institute of Automation (2003), "Database of 756 Grayscale Eye Images".

Cited at: http://www.sinobiometrics.com Version 1.0. 